\title{
Study on the cosmic ray intensity variation using scintillation counters for air shower observation
}

\section{Toshiyuki Nonaka, ${ }^{a, *}$ Akitoshi Oshima ${ }^{b}$ and Katsuya Yamazaki ${ }^{b}$ on behalf of the Telescope ArrayTelescope Array Collaboration}

(a complete list of authors can be found at the end of the proceedings)

${ }^{a}$ Institute for Cosmic Ray Research, University of Tokyo

5-1-5 Kashiwanoha Kashiwa, Chiba, Japan 277-8582

${ }^{b}$ College of Engineering, Chubu University, Kasugai, Aichi 487-8501, Japan

E-mail: nonaka@icrr.u-tokyo.ac.jp

\begin{abstract}
We report a study on the conversion of a giant cosmic ray observatory for air shower observation to observe cosmic ray intensity variations caused by solar activity, anisotropy associated with interplanetary disturbances, and detection of sudden cosmic ray events on the earth's surface. In this report, we use data from the surface detectors operated by the Telescope Array experiment located at $39^{\circ} \mathrm{N}, 112^{\circ} \mathrm{W}$ (total detector area: $2250 \mathrm{~m} 2$ ). In order to evaluate the cosmic ray intensity variations, we will compare the data with some of the corrections considered and with available world wide database such as Nagoya Muon detector and other observatories that have been in stable operation at different geographic longitudes. Finally, we will report on the intensity variations due to weather and solar activity recorded during the observation period.
\end{abstract}

$37^{\text {th }}$ International Cosmic Ray Conference (ICRC 2021)

July 12 th - 23rd, 2021

Online - Berlin, Germany

\footnotetext{
*Presenter
} 


\section{Introduction}

There exists a method to observe the changes in the state of interplanetary space due to solar activity through the observation of intensity variations and anisotropy of low energy galactic cosmic rays using ground-based cosmic ray instruments. $[1,2,3,4]$ The main muon instruments currently deployed are the Global Muon Detector Network (GMDN) group, the GRAPES-3 muon telescope in Tamil Nadu, India, and the Nagoya University Muon Telescope, which has been in stable operation for a long time. In monocular observations, the detection of interplanetary disturbances with fine structures, such as Loss Cone-type precursors [4], requires the estimation of intensity distributions in all longitude directions from the integration of 24 hours of data [5]. Therefore, quantitative detection of anisotropy is difficult during the passage of multiple interplanetary disturbances with high variability through the Earth. The purpose of this study is to operate the Telescope Array air shower instrument (TA), which is deployed over an area of about 700 square kilometers in the highlands of Utah, USA, as a muon instrument with a total area of 1500 square meters. The aim is to secure an observation point with high statistical accuracy just on the opposite side of the GRAPES-3 observation field of view. By doing so, we aim to reduce the detection limit of anisotropy from $0.5 \%$, which was the limit of previous studies $[5,6]$, and to detect amplitude fluctuations in real time.

For the purpose of this study written above, the following tasks (1) to (3) are necessary to convert
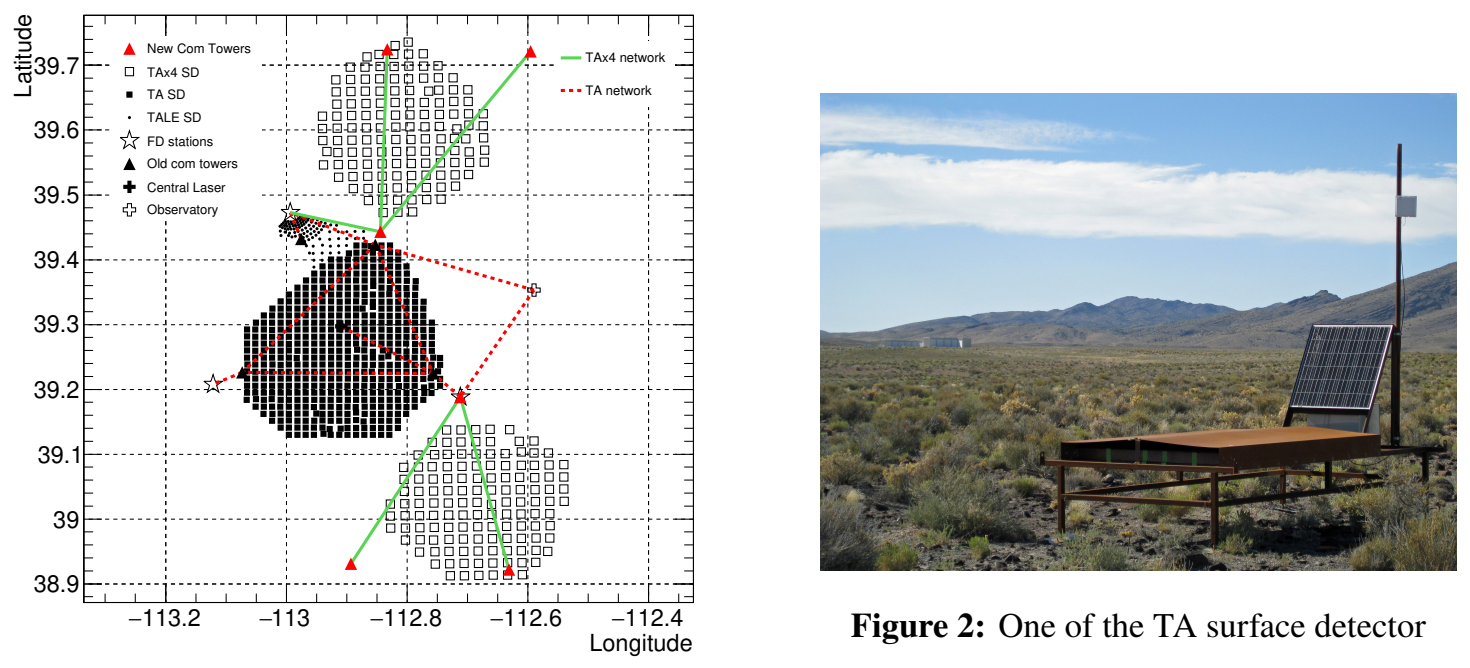

Figure 2: One of the TA surface detector

Figure 1: Entire long distance link between sites in TA-

TALE-TAx4 experiment.

the monitoring data of the air shower detector to data equivalent to the existing cosmic ray monitoring equipment.

(1) Record the meteorological conditions in the area where the instrument is deployed.

(2) Estimate the effect of atmospheric pressure and temperature on the particle count rate recorded by the instrument.

(3) Calculate the detector response as function of rigidity and asymptotic direction of primary 
cosmic ray.

(4) Comparing the variation of primary cosmic ray intensity estimated by the TA instrument with various instruments in different regions. This will confirm the reliability of the data.

\section{Experiment and analysis}

\subsection{Telescope Array Surface Detector}

The TA experiment is located in a desert area about 1,400 meters above sea level at 39.3 degrees north latitude and 112.9 degrees west longitude in Millard County, Utah, USA, about 200 kilometers southwest of Salt Lake City. The purpose of this experiment is to observe cascade showers caused by cosmic rays above $10^{19} \mathrm{eV}$. Figure 1 shows an overview of the TA surface particle detector and the experimental site.

The surface detector array consisting from 507 thin scintillation detectors with an effective area of $3 \mathrm{~m}^{2}$ per detector.

Fig.2 shows one of the TA surface detector. The detector is operated with solar panel and battery. Data is collected via wireless lan via control towers located at experimental site.

Atmospheric particles are recorded by the trigger at coincidence between two layer of scintillator. Here we call the trigger as Level-0 trigger. The threshold energy deposit at each layer is about $\sim 0.7 \mathrm{MeV}$. The rate of level- 0 trigger at each surface detector is about $\sim 750 \mathrm{~Hz}$. The distribution of integrated FADC count at each Level-0 trigger is recorded at each detector. Here we call this distribution as Mip histogram. The Mip histogram are collected every $10 \mathrm{~min}$ together with other monitor information including level-0 trigger rate. Minimum time resolution of level-0 trigger rate is $1 \mathrm{~min}$ average.
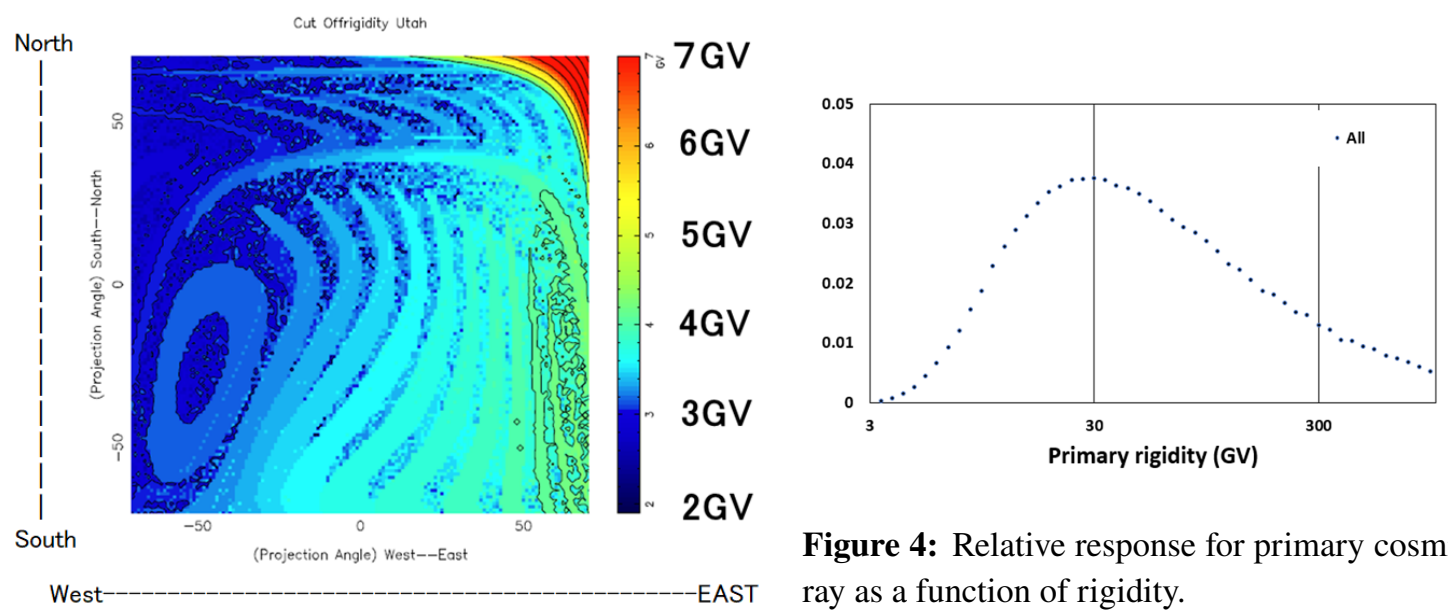

Figure 4: Relative response for primary cosmic ray as a function of rigidity.

Figure 3: Cut off rigidity at TA site.

\subsection{Detector response}

Using the CORSIKA air shower simulation code, primary cosmic rays are injected from the top of the atmosphere in the range of $2.5 \mathrm{GV}$ to $1000 \mathrm{GV}$ (zenith angle $<75^{\circ}$ to produce atmospheric 
particles at the observed height of TA.

Fig.3 shows cut off rigidity at TA site which is calculated independently. The cut off rigidity at the experimental site were calculated for various arriving direction of primary cosmic ray at top of atmosphere. The calculation were done with "backtracking method" assuming IGRF geomagnetic model(2000). Typically $3 \mathrm{GV}$ at vertical direction. Fig. 4 the response function to intensity variations of primary cosmic ray as a function of rigidity. We also estimated the directional distribution of sensitivity by back tracing the primary cosmic rays which generated atmospheric particles which generated trigger at our detector. In this way, we obtained the sensitivity distribution of our surface detector as a function of rigidity and asymptotic direction. The results are shown in Fig.3 and Fig.4.

\subsection{Data analysis}

Using the distribution of the energy deposit due to atmospheric particles obtained by TASD, here we call this distribution as Mip distribution. It is possible to obtain the count rate corrected for the variation of the trigger threshold due to the temperature change of the detector. The frequency of the energy loss distribution above half of its maximum value does not depend on the variation of the threshold. This count rate is denoted as N05. Since the shape of the energy loss distribution does not change over a long period of time, this can be used to analyze the following atmospheric correction independent of the detector gain variation. The following atmospheric correction can be analyzed independently of the detector gain variation.
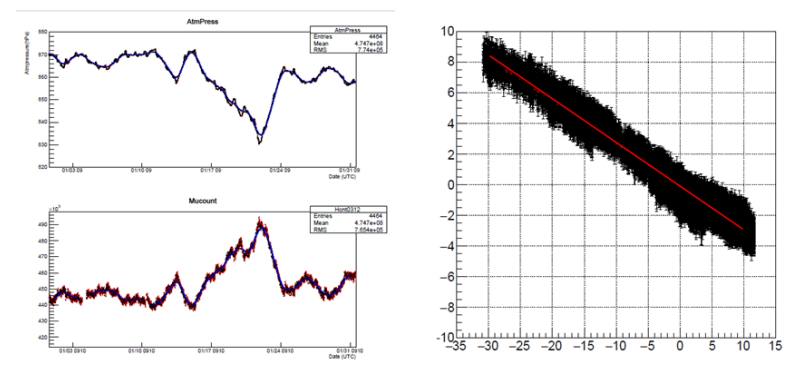

Figure 5: Pressure effect at TA SD. Left-Top: Atmospheric pressure variation for about sampled one month. LeftBottom Count rate of TASD after local temperature correction. Right: horizontal axis Pressure variation, vertical axis normalized count rate.

\subsection{Meteorological effect}

A-1: The counting variation of secondary particles due to changes in atmospheric pressure was investigated using the period of maximum pressure variation per month out of the 12-year observation period in Utah, USA. Fig. 5 shows the correlation between pressure change and the 
count rate. The pressure coefficient is estimated as $0.282 \pm 0.003 \% / h P a$

A-2: Counting changes due to changes in the upper atmosphere, where muons are produced, were investigated in Utah, USA, using real data and upper atmospheric data (GDAS data base), using the method described in [7] of the previous study. Fig.6 shows the correlation between $T_{m w}$ and the count rate. Table. 1 summarizes the coefficient obtained using a various parameter of atmosphere.

\section{Result and Summary}

We calculated characteristics of our observation such as response function and correction of local temperature effect. Also we considered atmospheric temperature effect and obtained data comparable with other observations in other geographical longitudes. After adapting these corrections, we compare our data with other observations of interplanetary disturbance event. Fig.7 shows a Forbush Decrease event which had been observed 2017. The effect of the correction for the effect of the upper atmosphere with and without the correction is shown.In this correction, we used the effective temperature weighted by the atmospheric density at each altitude, which corresponds to $T_{m w}$ in the table. 1 .

Figure 7: Summary of atmospheric temperature coefficient $\mathrm{H} 100 \mathrm{hPa}$ is altitude of $100 \mathrm{hPa}$. $T_{m w}$ is mass-weighted temperature. $T_{g r d}$ is ground temperature. $T_{16.5 \mathrm{~km}}$ is temperature at altitude $16.5 \mathrm{~km}$

\begin{tabular}{crr}
\hline \hline Method & Slope & Error \\
\hline$H 100 \mathrm{hPa}$ & $-3.992 \% / \mathrm{km}$ & $\pm 0.01 \% / \mathrm{km}$ \\
$T_{m w}$ & $-0.186 \% / \mathrm{K}$ & $\pm 0.005 \% / \mathrm{K}$ \\
$T_{g r d}$ & $-0.076 \% / \mathrm{K}$ & $\pm 0.002 \% / \mathrm{K}$ \\
$T_{16.5 \mathrm{~km}}$ & $+0.142 \% / \mathrm{K}$ & $\pm 0.001 \% / \mathrm{K}$ \\
\hline
\end{tabular}

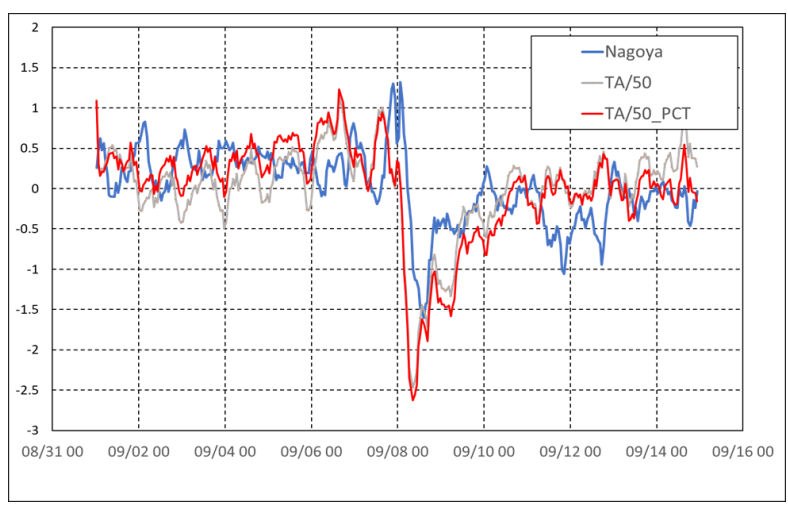

Figure 8: An forbush decrease event observed in Sep.2017.

Blue line shows variation observed Nagoya Muon Telescope. Gray line show TA surface detector data with local temperature and pressure correction. Red line shows TA surface detector data with local temperature, pressure and atmospheric temperature correction.

In the comparison between the vertical direction of Nagoya and the TA, the effect of temperature on 09/02-09/06 before the start of FD, and the effect of temperature around 09/14 were significant. The correction is about $0.3-4 \%$ in the larger cases. 


\section{Acknowledgements}

The Telescope Array experiment is supported by the Japan Society for the Promotion of Science(JSPS) through Grants-in-Aid for Priority Area 431, for Specially Promoted Research JP21000002, for Scientific Research (S) JP19104006, for Specially Promoted Research JP15H05693, for Scientific Research (S) JP15H05741 and JP19H05607, for Science Research (A) JP18H03705, for Young Scientists (A) JPH26707011, and for Fostering Joint International Research (B) JP19KK0074, by the joint research program of the Institute for Cosmic Ray Research (ICRR), The University of Tokyo; by the Pioneering Program of RIKEN for the Evolution of Matter in the Universe (r-EMU); by the U.S. National Science Foundation awards PHY-1404495, PHY-1404502, PHY-1607727, PHY-1712517, PHY-1806797 and PHY-2012934; by the National Research Foundation of Korea (2017K1A4A3015188, 2020R1A2C1008230, \& 2020R1A2C2102800) ; by the Ministry of Science and Higher Education of the Russian Federation under the contract 075-15-2020-778, RFBR grant 20-02-00625a (INR), IISN project No. 4.4501.18, and Belgian Science Policy under IUAP VII/37 (ULB). This work was partially supported by the grants ofThe joint research program of the Institute for Space-Earth Environmental Research, Nagoya University and Inter-University Research Program of the Institute for Cosmic Ray Research of University of Tokyo. The foundations of Dr. Ezekiel R. and Edna Wattis Dumke, Willard L. Eccles, and George S. and Dolores Doré Eccles all helped with generous donations. The State of Utah supported the project through its Economic Development Board, and the University of Utah through the Office of the Vice President for Research. The experimental site became available through the cooperation of the Utah School and Institutional Trust Lands Administration (SITLA), U.S. Bureau of Land Management (BLM), and the U.S. Air Force. We appreciate the assistance of the State of Utah and Fillmore offices of the BLM in crafting the Plan of Development for the site. Patrick A. Shea assisted the collaboration with valuable advice and supported the collaborations efforts. The people and the officials of Millard County, Utah have been a source of steadfast and warm support for our work which we greatly appreciate. We are indebted to the Millard County Road Department for their efforts to maintain and clear the roads which get us to our sites. We gratefully acknowledge the contribution from the technical staffs of our home institutions. An allocation of computer time from the Center for High Performance Computing at the University of Utah is gratefully acknowledged.

\section{References}

[1] T.Abu-Zayyad et al. Nuclear Instruments and Methods in Physics Research Section A 689, $87-97,2012$

[2] K. Munakata et al. Butsuri-Tansa Vol.65 No. 3 (2012) pp. 173-179

[3] Kozai et al. Earth, Planets and Space 66:151 (2014)

[4] K. Munakata, J. Geophys. Res., 105( A12), 27457- 27468

[5] K. Nagashima and K.Fujimoto et al.,Planet space sci (1992) 01109

[6] T. Nonaka PhD thesis Osaka City University (2005) 
[7] T. Nonaka et al. Proceedings of the 29th ICRC Vol.1 359-362 2005

[8] R. R. S Mendonca et al. Astrophysical Journal 830:88 (25pp) 2016 


\section{Full Author list: The Telescope Array Collaboration}

R.U. Abbasi ${ }^{1}$, T. Abu-Zayyad ${ }^{1,2}$, M. Allen ${ }^{2}$, Y. Arai ${ }^{3}$, R. Arimura ${ }^{3}$, E. Barcikowski ${ }^{2}$, J.W. Belz ${ }^{2}$, D.R. Bergman ${ }^{2}$, S.A. Blake ${ }^{2}$, I. Buckland ${ }^{2}$, R. $\mathrm{Cady}^{2}$, B.G. Cheon ${ }^{4}$, J. Chiba ${ }^{5}$, M. Chikawa ${ }^{6}$, T. Fujii ${ }^{7}$, K. Fujisue ${ }^{6}$, K. Fujita ${ }^{3}$, R. Fujiwara ${ }^{3}$, M. Fukushima ${ }^{6}$, R. Fukushima ${ }^{3}$, G. Furlich ${ }^{2}$, R. Gonzalez ${ }^{2}$, W. Hanlon ${ }^{2}$, M. Hayashi ${ }^{8}$, N. Hayashida ${ }^{9}$, K. Hibino ${ }^{9}$, R. Higuchi ${ }^{6}$, K. Honda ${ }^{10}$, D. Ikeda ${ }^{9}$, T. Inadomi ${ }^{11}$, N. Inoue ${ }^{12}$, T. Ishii ${ }^{10}$, H. Ito $^{13}$, D. Ivanov ${ }^{2}$, H. Iwakura ${ }^{11}$, A. Iwasaki ${ }^{3}$, H.M. Jeong ${ }^{14}$, S. Jeong ${ }^{14}$, C.C.H. Jui ${ }^{2}$, K. Kadota ${ }^{15}$, F. Kakimoto ${ }^{9}$, O. Kalashev ${ }^{16}$, K. Kasahara ${ }^{17}$, S. Kasami ${ }^{18}$, H. Kawai ${ }^{19}$, S. Kawakami ${ }^{3}$, S. Kawana ${ }^{12}$, K. Kawata ${ }^{6}$, I. Kharuk ${ }^{16}$, E. Kido ${ }^{13}$, H.B. Kim ${ }^{4}$, J.H. Kim ${ }^{2}$, J.H. Kim ${ }^{2}$, M.H. Kim ${ }^{14}$, S.W. Kim ${ }^{14}$, Y. Kimura ${ }^{3}$, S. Kishigami ${ }^{3}$, Y. Kubota ${ }^{11}$, S. Kurisu ${ }^{11}$, V. Kuzmin ${ }^{16^{*}}$, M. Kuznetsov ${ }^{16,20}$, Y.J. Kwon ${ }^{21}$, K.H. Lee ${ }^{14}$, B. Lubsandorzhiev ${ }^{16}$, J.P. Lundquist ${ }^{2,22}$, K. Machida ${ }^{10}$, H. Matsumiya ${ }^{3}$, T. Matsuyama ${ }^{3}$, J.N. Matthews ${ }^{2}$, R. Mayta ${ }^{3}$, M. Minamino ${ }^{3}$, K. Mukai ${ }^{10}$, I. Myers ${ }^{2}$, S. Nagataki ${ }^{13}$, K. Nakai $^{3}$, R. Nakamura ${ }^{11}$, T. Nakamura ${ }^{23}$, T. Nakamura ${ }^{11}$, Y. Nakamura ${ }^{11}$, A. Nakazawa ${ }^{11}$, E. Nishio ${ }^{18}$, T. Nonaka ${ }^{6}, \mathrm{H}_{\text {. Oda }}{ }^{3}, \mathrm{~S}_{\text {O Ogio }}{ }^{3,24}$, M. Ohnishi ${ }^{6}$, H. Ohoka ${ }^{6}$, Y. Oku ${ }^{18}$, T. Okuda ${ }^{25}$, Y. Omura ${ }^{3}$, M. Ono ${ }^{13}$, R. Onogi ${ }^{3}$, A. Oshima ${ }^{3}$, S. Ozawa ${ }^{26}$, I.H. Park ${ }^{14}$, M. Potts ${ }^{2}$, M.S. Pshirkov ${ }^{16,27}$, J. Remington ${ }^{2}$, D.C. Rodriguez ${ }^{2}$, G.I. Rubtsov ${ }^{16}$, D. $\mathrm{Ryu}^{28}$, H. Sagawa ${ }^{6}$, R. Sahara ${ }^{3}$, Y. Saito ${ }^{11}$, N. Sakaki ${ }^{6}$, T. Sako ${ }^{6}$, N. Sakurai ${ }^{3}$, K. Sano $^{11}$, K. Sato ${ }^{3}$, T. Seki ${ }^{11}$, K. Sekino ${ }^{6}$, P.D. Shah ${ }^{2}$, Y. Shibasaki ${ }^{11}$, F. Shibata ${ }^{10}$, N. Shibata ${ }^{18}$, T. Shibata ${ }^{6}$, H. Shimodaira ${ }^{6}$, B.K. Shin ${ }^{28}$, H.S. Shin ${ }^{6}$, D. Shinto ${ }^{18}$, J.D. Smith ${ }^{2}$, P. Sokolsky ${ }^{2}$, N. Sone ${ }^{11}$, B.T. Stokes ${ }^{2}$, T.A. Stroman ${ }^{2}$, Y. Takagi ${ }^{3}$, Y. Takahashi ${ }^{3}$, M. Takamura $^{5}$, M. Takeda $^{6}$, R. Takeishi ${ }^{6}$,

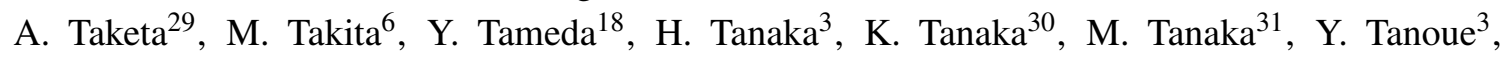
S.B. Thomas ${ }^{2}$, G.B. Thomson ${ }^{2}$, P. Tinyakov ${ }^{16,20}$ I. Tkachev $^{16}$, H. Tokuno ${ }^{32}$, T. Tomida ${ }^{11}$, S. Troitsky ${ }^{16}$, R. Tsuda ${ }^{3}$, Y. Tsunesada ${ }^{3,24}$, Y. Uchihori ${ }^{33}$, S. Udo ${ }^{9}$, T. Uehama ${ }^{11}$, F. Urban ${ }^{34}$, T. Wong ${ }^{2}$, K. Yada $^{6}$, M. Yamamoto ${ }^{11}$, K. Yamazaki ${ }^{9}$, J. Yang ${ }^{35}$, K. Yashiro ${ }^{5}$, F. Yoshida $^{18}$, Y. Yoshioka ${ }^{11}$, Y. Zhezher ${ }^{6,16}$, and Z. Zundel ${ }^{2}$

${ }^{1}$ Department of Physics, Loyola University Chicago, Chicago, Illinois, USA

${ }^{2}$ High Energy Astrophysics Institute and Department of Physics and Astronomy, University of Utah, Salt Lake City, Utah, USA

${ }^{3}$ Graduate School of Science, Osaka City University, Osaka, Osaka, Japan

${ }^{4}$ Department of Physics and The Research Institute of Natural Science, Hanyang University, Seongdong-gu, Seoul, Korea

${ }^{5}$ Department of Physics, Tokyo University of Science, Noda, Chiba, Japan

${ }^{6}$ Institute for Cosmic Ray Research, University of Tokyo, Kashiwa, Chiba, Japan

7 The Hakubi Center for Advanced Research and Graduate School of Science, Kyoto University, Kitashirakawa-Oiwakecho, Sakyo-ku, Kyoto, Japan

${ }^{8}$ Information Engineering Graduate School of Science and Technology, Shinshu University, Nagano, Nagano, Japan

${ }^{9}$ Faculty of Engineering, Kanagawa University, Yokohama, Kanagawa, Japan

${ }^{10}$ Interdisciplinary Graduate School of Medicine and Engineering, University of Yamanashi, Kofu, Yamanashi, Japan

${ }^{11}$ Academic Assembly School of Science and Technology Institute of Engineering, Shinshu University, Nagano, Nagano, Japan 
12 The Graduate School of Science and Engineering, Saitama University, Saitama, Saitama, Japan

${ }^{13}$ Astrophysical Big Bang Laboratory, RIKEN, Wako, Saitama, Japan

${ }^{14}$ Department of Physics, SungKyunKwan University, Jang-an-gu, Suwon, Korea

${ }^{15}$ Department of Physics, Tokyo City University, Setagaya-ku, Tokyo, Japan

${ }^{16}$ Institute for Nuclear Research of the Russian Academy of Sciences, Moscow, Russia

${ }^{17}$ Faculty of Systems Engineering and Science, Shibaura Institute of Technology, Minato-ku, Tokyo, Japan

18 Department of Engineering Science, Faculty of Engineering, Osaka Electro-Communication University, Neyagawa-shi, Osaka, Japan

${ }^{19}$ Department of Physics, Chiba University, Chiba, Chiba, Japan

${ }^{20}$ Service de Physique Theorique, University Libre de Bruxelles, Brussels, Belgium

${ }^{21}$ Department of Physics, Yonsei University, Seodaemun-gu, Seoul, Korea

${ }^{22}$ Center for Astrophysics and Cosmology, University of Nova Gorica, Nova Gorica, Slovenia

${ }^{23}$ Faculty of Science, Kochi University, Kochi, Kochi, Japan

${ }^{24}$ Nambu Yoichiro Institute of Theoretical and Experimental Physics, Osaka City University, Osaka, Osaka, Japan

${ }^{25}$ Department of Physical Sciences, Ritsumeikan University, Kusatsu, Shiga, Japan

${ }^{26}$ Quantum ICT Advanced Development Center, National Institute for Information and Communications Technology, Koganei, Tokyo, Japan

${ }^{27}$ Sternberg Astronomical Institute, Moscow M.V. Lomonosov State University, Moscow, Russia

${ }^{28}$ Department of Physics, School of Natural Sciences, Ulsan National Institute of Science and Technology, UNIST-gil, Ulsan, Korea

${ }^{29}$ Earthquake Research Institute, University of Tokyo, Bunkyo-ku, Tokyo, Japan

${ }^{30}$ Graduate School of Information Sciences, Hiroshima City University, Hiroshima, Hiroshima, Japan

${ }^{31}$ Institute of Particle and Nuclear Studies, KEK, Tsukuba, Ibaraki, Japan

32 Graduate School of Science and Engineering, Tokyo Institute of Technology, Meguro, Tokyo, Japan

${ }^{33}$ Department of Research Planning and Promotion, Quantum Medical Science Directorate, National Institutes for Quantum and Radiological Science and Technology, Chiba, Chiba, Japan

${ }^{34}$ CEICO, Institute of Physics, Czech Academy of Sciences, Prague, Czech Republic

${ }^{35}$ Department of Physics and Institute for the Early Universe, Ewha Womans University, Seodaaemungu, Seoul, Korea 